\title{
Quaderni
}

QUADERNI Communication, technologies, pouvoir

\section{Idées, institutions politiques et production de l'expertise : une perspective comparative sur le rôle des think tanks au Canada et aux États-Unis}

Daniel Béland

\section{OpenEdition}

Journals

Édition électronique

URL : http://journals.openedition.org/quaderni/505

DOI : 10.4000/quaderni.505

ISSN : 2105-2956

Éditeur

Les éditions de la Maison des sciences de l'Homme

\section{Édition imprimée}

Date de publication : 5 octobre 2009

Pagination : 39-48

Référence électronique

Daniel Béland, «Idées, institutions politiques et production de l'expertise : une perspective comparative sur le rôle des think tanks au Canada et aux États-Unis », Quaderni [En ligne], 70 | Automne 2009, mis en ligne le 05 octobre 2011, consulté le 02 mai 2019. URL : http:// journals.openedition.org/quaderni/505; DOI : 10.4000/quaderni.505 


\section{$D$ ossier}

\section{idées,}

institutions politiques

et production

de l'expertise :

une perspective comparative sur

le rôle des think

tanks au Canada

et aux États-Unis'

\section{Daniel \\ Béland}

Titulaire de la Chaire de Recherche en politiques publiques Université de la Saskatchewan Canada
Depuis les années 1990, un nombre grandissant de chercheurs s'intéresse au rôle des idées dans l'élaboration des politiques publiques ${ }^{2}$. Simultanément, dans le monde anglophone en particulier, les publications sur le rôle des « boîtes à idées » (think tanks) dans la production de l'expertise en matière de politiques publiques se multiplient ${ }^{3}$. Malheureusement, ces deux littératures sont rarement discutées côte à côte dans les travaux de sciences humaines.

L'objectif de cet article est précisément d'intégrer ces deux littératures afin de mieux saisir le rôle des think tanks dans la dissémination des idées et celui de l'expertise dans le champ des politiques publiques. Après une discussion théorique consacrée aux rapports entre idées, institutions politiques et construction de l'expertise, l'article propose une brève analyse comparative du rôle des think tanks aux États-Unis et au Canada. L'objectif de cette comparaison est d'expliquer pourquoi les think tanks jouent un rôle politique plus proéminent aux États-Unis qu'au Canada. Tel que démontré, les institutions politiques américaines favorisent la multiplication des think tanks aux États-Unis. Au Canada, des institutions comme le Bureau du Conseil privé fédéral limite, au contraire, l'intérêt de l'État pour les think tanks, ce dernier ayant développé, au fil des années, une grande capacité d'expertise. Une telle situation n'empêche cependant pas les think tanks de jouer un rôle significatif dans l'espace médiatique et politique canadien. Au-delà des différences observées entre le Canada et les ÉtatsUnis, ces deux pays - on le verra - connaissent en outre une véritable polarisation de l'expertise inséparable de la montée en puissance des think tanks néo-conservateurs, et cela depuis le début 
des années 1970.

\section{Pourquoi se tourner vers l'analyse des idées?}

Depuis les années 1990, en réaction aux approches strictement institutionnalistes ou matérialistes du politique, plusieurs sociologues et politologues ont décidé de mettre désormais l'accent sur le rôle des idées dans le domaine des politiques publiques. Bien que ces auteurs ne s'entendent pas toujours sur la terminologie à adopter en la matière (《 idées », « idéologies », 《 paradigmes », « discours », 《 référentiels »), ils partagent généralement un même constat : les approches réductionnistes comme le matérialisme historique et les théories du « choix rationnel» si populaires aux États-Unis ne rendent compte ni de la complexité ni de la construction du sens dans le développement des politiques publiques ${ }^{4}$. Quant aux approches institutionnalistes classiques, elles ont l'avantage de mettre l'accent sur le phénomène de la contingence en tant qu'il est inséparable du caractère proprement historique des institutions politiques. Toutefois, comme le remarquent des sociologues tels que John Campbell et François-Xavier Merrien, en soi, les approches institutionnelles peinent à expliquer les changements parfois brusques qui surviennent dans un secteur particulier d'intervention de l'État sinon même dans la société en général ${ }^{5}$. Bien que des facteurs économiques puissent expliquer certains de ces changements, des auteurs comme Mark Blyth et Craig Parsons ont démontré qu'il était indispensable d'étudier de plus près les idées relayées par certains acteurs sociaux pour comprendre leurs actions et leurs choix politiques ${ }^{6}$, sans négliger bien entendu le fait que, sauf crise majeure, les institutions politiques et les pro- grammes préexistants témoignent de contraintes toujours susceptibles d'influencer directement les décisions de ces mêmes acteurs. Il convient enfin de noter - on y reviendra - que les institutions propres à chaque pays conditionnent largement le développement de l'expertise et la diffusion de nouvelles idées et propositions de réforme dans le champ des politiques publiques? ${ }^{7}$.

\section{Comment les idées peuvent-elles influencer le développement des politiques publiques?}

Les idées informent le champ des politiques publiques de trois principales manières ${ }^{8}$. Tout d'abord, les experts, journalistes, mouvements sociaux et parfois les élus eux-mêmes participent à la construction des questions de politiques publiques en définissant les problèmes économiques et sociaux nécessitant une intervention de l'État. Depuis des décennies, plusieurs chercheurs ont ainsi décrit les multiples mécanismes permettant à certains acteurs d'influencer, directement ou indirectement, les citoyens et les politiques en attirant leur attention sur des problèmes présentés comme urgents ${ }^{9}$. Cette attention se limitant forcément à un nombre restreint d'enjeux et de problématiques clés, on assiste dès lors à une concurrence particulièrement aiguisée - et cela même si elle est implicite - entre divers groupes et acteurs sociaux cherchant à inscrire à l'agenda politique des questions aussi diverses que la délinquance juvénile, l'immigration clandestine ou encore la violence conjugale. À côté des ressources financières et des réseaux politiques propres à ces acteurs, leur capacité même à convaincre les citoyens et les élus de la nécessité de prendre ces problèmes au sérieux et de les traiter en priorité se révèle être un aspect fondamental de l'élaboration 
des politiques publiques. Avant de discuter les choix politiques qui s'offrent à eux pour résoudre certains problèmes, les responsables publics doivent ainsi s'entendre sur les questions exigeant toute leur attention; la définition des problèmes et leur mise à l'agenda s'inscrivant, par conséquent, en amont des processus législatifs directement à l'origine de nouvelles mesures dans le domaine des politiques publiques.

Les acteurs politiques se doivent ensuite d'examiner les différentes options à même de répondre aux problématiques concernées; le nombre et l'orientation des options examinées étant très souvent conditionnés par les prédispositions idéologiques des responsables publics mais aussi par le profil des experts convoqués pour l'occasion. Outre des positionnements idéologiques généraux associés à des antagonismes doctrinaux traditionnels (i.e. gauche/droite, progressisme/ conservatisme), les acteurs politiques et leurs experts-conseillers sont également susceptibles de partager un paradigme plus technique. On peut donc définir, avec Peter Hall, un paradigme de politique publique comme un cadre analytique structuré qui « spécifie non seulement les objectifs d'une politique et le type d'instruments qui peut être utilisé pour les atteindre mais [aussi] la nature même des problèmes qui doivent être affrontés $»^{10}$. Selon ce politologue, il existe ainsi un lien direct entre la «définition » même des problématiques et l'élaboration de « solutions » dans le domaine des politiques publiques. Il faut cependant remarquer que, dans bien des cas, les responsables ont en tête des «solutions toutes faites » qu'ils s'efforcent par la suite d'arrimer aux nouveaux problèmes de l'heure. Depuis les années 1980, par exemple, les tenants du para- digme néolibéral s'efforcent de jumeler un « problème » inscrit à l'agenda politique de nombreux pays comme le vieillissement démographique avec une « solution » libérale traditionnelle telle que l'épargne individuelle qui, sous le nom de " prévoyance », était pourtant déjà vantée il y a un siècle de cela par les libéraux d'alors, lesquels s'opposaient à l'époque à la création des premiers régimes publics de retraite ${ }^{11}$. Dans cette perspective, le champ des options disponibles face à un problème précis est largement déterminé par des considérations idéologiques générales dont l'histoire est parfois bien antérieure à l'inscription de ce problème à l'agenda politique.

Les idées constituent enfin une arme politique permettant à certains acteurs de justifier, auprès de l'opinion publique, des groupes de pression ou encore d'autres responsables, l'adoption d'une série de mesures. On peut alors parler d'un discours idéologique orienté vers ce que Robert Cox qualifie de « construction sociale de la nécessité de réformer» (social construction of the need to reform $)^{12}$, les politiques se devant de légitimer tout à la fois les mesures à adopter et le besoin lui-même de " faire quelque chose » pour répondre aux problèmes identifiés. Les idées et le discours politique qui les relaye participent alors à la construction d'impératifs idéologiques susceptibles de rendre inéluctable une réforme, en apparence du moins. Pour justifier ainsi une éventuelle réduction des prestations sociales, des élus se réfèreront tantôt à un processus institutionnel historique tel que la construction européenne, tantôt à un mot d'ordre plus général comme celui de « compétitivité économique $»^{13}$. Il est par ailleurs intéressant de noter que dans les pays où les employeurs et les syndicats jouent un 
rôle institutionnel primordial dans la conception des politiques publiques, le discours justificatif s'adresse souvent bien plus aux «partenaires sociaux » qu'à l'opinion publique en général, ce qui n'est pas le cas là où ces mêmes acteurs ne sont pas formellement intégrés au processus de décision ${ }^{14}$. Cette dernière remarque illustrant, une fois encore, le rôle particulièrement déterminant des institutions politiques dans la diffusion des idées et le développement historique des think tanks.

\section{Le rôle des think tanks dans la production de l'expertise et la diffusion des idées}

$\mathrm{Au}$ sens strict du terme, think tank signifie « réservoir intellectuel $»$. Aucune traduction française n'est toutefois satisfaisante. Situé au carrefour de la recherche et de l'action politique, le think tank est une organisation de la société civile qui se donne généralement une mission pragmatique : éclairer voire influencer les citoyens, les élus ou encore les groupes de pression au sujet des grandes questions de politique publique. Qu'il soit généraliste ou spécialisé dans des domaines comme la politique étrangère ou les politiques économiques, le think tank se situe en quelque sorte à la frontière de l'expertise et de l'action politique. Cette position si singulière s'avère déterminante lorsqu'il s'agit précisément de penser les relations s'établissant entre think tanks, circulation des idées et discours de légitimation. Dans un pays comme les États-Unis où ils occupent une position privilégiée au sein du champ du pouvoir, les « boîtes à idées » ont notamment pour fonction celle de médiatiser les idées en vogue émanant des milieux universitaires et intellectuels en les adaptant aux exigences de l'action publique, et cela notamment en les rendant accessibles aux fonctionnaires, aux élus ou encore aux journalistes. Les think tanks peuvent ainsi jouer, en matière de politiques publiques, un rôle crucial dans la diffusion des trois grandes catégories d'idées précédemment mentionnés.

Les think tanks sont premièrement en mesure de promouvoir l'étude de certaines problématiques socio-économiques, tout en s'efforçant parfois d'imposer un cadrage bien précis à ces questions. $\mathrm{Au}$ Canada comme aux États-Unis - pour ne prendre qu'un exemple particulièrement emblématique -, un certain nombre de think tanks ont ainsi décidé, depuis plusieurs années maintenant, de s'immiscer dans le débat relatif à l'impact supposé du vieillissement démographique sur les régimes de retraites ${ }^{15}$.

Les experts travaillant pour divers think tanks peuvent ensuite offrir leurs conseils aux élus et aux fonctionnaires, parfois de façon directe, lors de conférences, et souvent de manière indirecte, à travers des rencontres et des communications informelles. On peut considérer dans cette perspective, sans risque d'être démenti, que l'industrie privée de la consultance participe alors, de facto, à la conception de certaines réformes ou qu'elle tente, tout au moins, d'influencer le débat à la fois technique et politique concernant les « solutions » à apporter aux «problèmes » inscrits - ou susceptibles d'être inscrits - à l'agenda.

Dans certains pays, enfin, il est des think tanks qui n'hésitent pas à s'introduire directement dans l'arène politique pour légitimer des décisions ou des projets de loi tout à fait précis. Ces organisations de la société civile peuvent explicitement 
jouer un rôle idéologique sinon même clairement partisan, la stratégie adoptée les métamorphosant ainsi en véritables "entrepreneurs politiques » capables de transcender l'opposition que Max Weber décrivait, il y a de cela un siècle, entre « savant » et « politique $»{ }^{16}$. L'expert, il est vrai, est autant sinon davantage un « politique » qu'un « savant» pour certains think tanks, sa mission essentielle se résumant alors à participer ouvertement à la lutte politique, et cela en utilisant autant les outils traditionnels de la propagande que les statistiques et autres « rapports de recherche ». Cette logique dominante de politisation de l'industrie de la consultance semble bien s'être développée, aux États-Unis et au Canada notamment, avec la polarisation croissante de l'expertise privée.

\section{Institutions politiques et influence comparée des think tanks aux États-Unis et au Canada}

Il est impossible de comprendre le développement des think tanks aux États-Unis sans prendre en compte une caractéristique majeure du système politique américain: sa fragmentation renvoyant à l' « équilibre des pouvoirs », un dispositif à l'origine destiné à prévenir une concentration par trop excessive du pouvoir politique. L'équilibre des pouvoirs se définit à la fois comme une séparation et une immixtion de l'exécutif, du législatif et du judiciaire, ces niveaux de pouvoir s'incarnant dans des institutions théoriquement concurrentes les unes des autres ${ }^{17}$. Bien que cette caractéristique générale du système politique américain ne puisse en tant que telle expliquer l'émergence et la multiplication des think tanks aux États-Unis, la fragmentation des institutions politiques - plus encore que le fédéralisme - engendre néanmoins une multiplication des demandes d'expertise, ce qui a pour effet de stimuler l'essor des « boîtes à idées » et autres organisations de recherche nées de la société civile ${ }^{18}$.

Pour être tout à fait précis, sur le plan fédéral, deux traits essentiels du système politique américain rendent compte du développement des think tanks au sein de cette société : la perméabilité et l'éclatement de l'administration fédérale, d'une part, le manque de cohésion idéologique des partis politiques actifs au plan national, de l'autre ${ }^{19}$. L'administration fédérale, premièrement, se caractérise par sa fragmentation, son sous-développement en comparaison à l'administration de certains États européens et, plus important encore, par sa relative perméabilité à l'égard de ladite société civile. Ainsi, plusieurs hauts fonctionnaires font d'abord carrière dans le secteur privé, l'université ou le monde des think tanks avant de rejoindre les rangs de la haute fonction publique ; le phénomène inverse étant également fréquent à savoir l'embauche d'anciens responsables politiques par les grandes entreprises privées et les plus influents think tanks. De plus, face aux insuffisances de l'administration, le recours à des experts indépendants s'avère souvent être une nécessité, l'État fédéral faisant ainsi régulièrement appel à des consultants extérieurs pour analyser certaines problématiques et proposer, par la même occasion, des réponses à des questions de politique publique.

L'absence d'une discipline de parti aux ÉtatsUnis est la seconde condition favorable à la multiplication des think tanks dans ce pays. Jouissant généralement d'une certaine autonomie idéologique face à l'appareil du 
parti, les membres du Congrès, comme ceux d'ailleurs de l'exécutif, font régulièrement appel à des consultants extérieurs travaillant dans des «boîtes à idées" lorsque vient le temps, par exemple, d'élaborer un projet de loi. Perméable à l'influence des groupes de pression, le Congrès s'ouvre lui aussi à l'industrie des think tanks et à ses experts accrédités.

Durant la majeure partie du vingtième siècle, ces conditions institutionnelles - couplées à l'expansion du rôle de l'État et au culte des experts du New Deal et du keynésianisme des années 50 et 60 - vont, à l'évidence, favoriser l'émergence des premiers grands think tanks américains tels que le Brookings Institution (1927), la Rand Foundation (1948) et l'Urban Institute $(1968)^{20}$. Alors que la première de ces organisations est, à l'origine, conçue comme une «université sans étudiants» effectuant des recherches indépendantes financées par de puissantes fondations charitables, les deux dernières s'efforcent, quant à elles, d'obtenir des contrats de recherche auprès de diverses instances gouvernementales et parastatales. Dans le courant des années 1970, ce sont alors des think tanks néo-conservateurs comme l'Heritage Foundation $(1973)^{21}$ et le Cato Institute (1977) $)^{22}$ qui sont apparus sur la scène politique, favorisant une polarisation idéologique de l'expertise, et participant ouvertement aux croisades d'une droite conservatrice désireuse de réformer certaines politiques publiques, et cela notamment dans le domaine social ${ }^{23}$. Enfin, au cours des deux dernières décennies - en réaction à cette multiplication d'organisations conservatrices - des think tanks de gauche tels que le Center for American Progress (2003) ${ }^{24}$ sont venus eux aussi accentuer cette polarisation de l'expertise, sans réussir pour autant à chasser de leur position dominante, au plan fédéral comme au niveau des États fédérés ${ }^{25}$, les plus influents think tanks néo-conservateurs. Il demeure cependant une réalité incontestable : bien qu'il ne soit pas toujours facile de mesurer leur influence réelle, les think tanks progressistes comme néo-conservateurs jouent un rôle idéologique majeur dans la démocratie américaine, en raison notamment des conditions institutionnelles précédemment évoquées.

L'examen du cas canadien vient, à son tour, confirmer l'importance des institutions politiques dans la médiation de l'expertise et celle des think tanks dans l'élaboration d'une véritable politique des idées. Si elle est bien présente dans les médias et le débat public, l'industrie privée de la consultance joue en effet le plus souvent, au Canada, un rôle moins actif dans le développement des politiques publiques que ce que l'on a pu observer aux États-Unis. Des différences institutionnelles majeures entre ces deux pays - cela a été dit - expliquent en grande partie ce contraste ${ }^{26}$. Au Canada, l'État fédéral dispose ainsi de moyens d'expertise plus importants et centralisés qu'aux États-Unis, songeons notamment à ce centre intellectuel du gouvernement fédéral qu'est le Bureau du Conseil privé (Privy Council Office), influente et prestigieuse institution animée par des équipes de recherche composées de fonctionnaires au fait des plus récents travaux universitaires. Audelà de cette institution, de nombreux experts travaillent d'ailleurs exclusivement pour le compte de l'État fédéral ou de l'une des dix provinces canadiennes, et au lieu de se déplacer 
régulièrement entre les secteurs privé et public - comme c'est notamment le cas aux États-Unis - les hauts fonctionnaires font, pour la grande majorité d'entre eux, l'essentiel de leur carrière professionnelle au sein de l'appareil d'État. La discipline de parti aux parlements fédéral et provinciaux est un autre facteur institutionnel majeur, cette discipline rendant les élus beaucoup plus dépendants envers leur formation politique que ne le sont leurs homologues américains. Finalement, compte tenu de leur position institutionnelle plus défavorable, les think tanks canadiens peuvent rarement bénéficier des ressources financières et intellectuelles aussi importantes que celles dont jouissent les grandes organisations américaines comme le Brookings Institution et l'Heritage Foundation. Qui plus est, en raison de leur prestige limité, de leur manque relatif de moyens et des excellentes conditions de travail offertes par les grandes universités du pays, les think tanks nationaux sont dans une situation moins enviable que celle des principales « boîtes à idées » des États-Unis pour embaucher des experts hautement qualifiés et détenteurs d'un doctorat ${ }^{27}$.

Au Canada, la conjonction des facteurs institutionnels décrits ci-dessus tend, par conséquent, à limiter l'influence des quatre principaux think tanks nationaux en activité : le C.D. Howe Institute, le Conference Board of Canada (CBC), le Fraser Institute et l'Institute for Research on Public Policy (IRPP) $)^{28}$. Il serait cependant faux d'affirmer que les situations américaine et canadienne n'ont absolument rien en commun. Premièrement, dans certaines circonstances, les think tanks canadiens peuvent eux aussi exercer une influence politique majeure, les obstacles institutionnels n'étant pas toujours aussi insurmontables qu'on pourrait le croire, notamment en raison du rayonnement de ces organisations sur Internet, dans les médias traditionnels et dans les réseaux de politiques publiques ${ }^{29}$. Deuxièmement, l'attitude explicitement idéologique - sinon même ouvertement partisane - de think tanks comme le très néolibéral Fraser Institute a favorisé au Canada une polarisation de l'expertise semblable à celle que l'on peut observer aux États-Unis ; le Fraser Institute s'inspirant d'ailleurs des diverses méthodes employées, depuis les années 1970, par des «boîtes à idées » néo-conservatrices américaines comme le CATO Institute. Il existe ainsi une convergence idéologique et une influence transfrontalière exercée par les think tanks américains sur leurs homologues canadiens qui mériterait très probablement, à elle seule, une étude approfondie. Quoiqu'il en soit, et en dépit même de ces rapprochements d'ordre idéologique, le poids des institutions nationales semble expliquer, dans une très large mesure, les profondes différences observées, entre les deux pays, dans le domaine de la production de l'expertise et de la diffusion des idées.

\section{Conclusion}

À l'avenir, il serait intéressant d'analyser l'impact de ces différences institutionnelles sur la nature et l'évolution récente de la politique des idées aux États-Unis et au Canada. Une telle analyse pourrait, en outre, examiner de plus près l'une des principales conclusions de la présente contribution, à savoir l'existence d'un lien quasiment organique entre, d'une part, les think tanks et leurs pratiques d'expertise, et de l'autre, 
le rôle crucial des idées dans l'élaboration des politiques publiques. Les chercheurs en sciences humaines seraient, par ailleurs, fort inspirés s'ils mettaient plus systématiquement en relation la littérature consacrée aux think tanks avec celle relative aux idées dans le domaine des politiques publiques, et cela en gardant constamment à l'esprit l'influence, parfois déterminante, des institutions politiques sur la production de l'expertise et la diffusion des idées.
$\mathrm{N}$

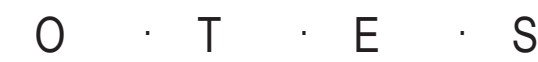

1. L'auteur tient à remercier Geoffrey Geuens et André Lecours pour leurs commentaires et suggestions.

2. Daniel Béland, «Ideas and Social Policy: An Institutionalist Perspective », dans Social Policy \& Administration, $\mathrm{n}^{\circ} 39$ (1), 2005, pp. 1-18; Bruno Palier et Yves Surel, «Les “trois I" et l'analyse de l'État en action », dans Revue française de science politique, $\mathrm{n}^{\circ} 55(1), 2005$, pp. 7-32

3. James McGann et R. Kent Weaver (dir.), Think Tanks and Civil Societies, Piscataway, Transaction Publishers, 2000 ; Diane Stone et Andrew Denham (dir.), Think Tank Traditions: Policy Research and the Politics of Ideas, Manchester, Manchester University Press, 2004 ; Diane Stone, Andrew Denham et Mark Garnett (dir.), Think Tanks across Nations: A Comparative Approach, Manchester, Manchester University Press, 1998.

4. Frank Fischer, Reframing Public Policy: Discursive Politics and Deliberative Practices, Oxford, Oxford University Press, 2003.

5. John L. Campbell, Institutional Change and Globalization, Princeton, Princeton University Press, 2004 ; François-Xavier Merrien, L'État-providence, Paris, Presses Universitaires de France, 1997.

6. Mark Blyth, Great Transformations: Economic Ideas and Institutional Change in the Twentieth Century, Cambridge, Cambridge University Press, 2002 ; Craig Parsons, A Certain Idea of Europe, Ithaca, Cornell University Press, 2003.

7. John L. Campbell et Ove Kaj Pedersen, « Knowledge Regimes and the Varieties of Capitalism », dans Daniel Béland et Robert H. Cox (dir.), Ideas and Politics in Social Science Research, New York, Oxford University Press, à paraître.

8. John W. Kingdon, Agendas, Alternatives, and Public 
Policies (deuxième édition), New York, Harper Collins, 1995 (1984).

9. Deborah Stone, Policy Paradox: The Art of Political Decision Making, New York, W.W. Norton, 1997.

10. Peter A. Hall, « Policy Paradigms, Social Learning and the State: The Case of Economic Policymaking in Britain », dans Comparative Politics, n²5(3), 1993, p. 279.

11. Robert Castel, Les métamorphoses de la question sociale, Paris, Fayard, 1995 ; Bruno Dumons et Gilles Pollet, L'État et les retraites, Paris, Belin, 1990 ; Henri Hatzfeld, Du paupérisme à la sécurité sociale 1850-1940, Nancy, Presses Universitaires de Nancy, 1989 (1971).

12. Robert H. Cox, «The Social Construction of an Imperative: Why Welfare Reform Happened in Denmark and the Netherlands but Not in Germany », dans World Politics, n53(3), 2001, pp. 463-498.

13. Colin Hay et Ben Rosamond, "Globalization, European Integration and the Discursive Construction of Economic Imperatives », dans Journal of European Public Policy, ${ }^{\circ}$ 9(2), 2002, pp. 147-167.

14. Vivien A. Schmidt, The Futures of European Capitalism, Oxford, Oxford University Press, 2002.

15. Daniel Béland, « Expertise et politique des retraites: l'influence des think tanks aux États-Unis », dans L'Année de la régulation, $\mathrm{n}^{\circ} 4,2000$, pp. 74-99. Pour une comparaison entre la France et les États-Unis concernant la production de l'expertise et la question du vieillissement, on lira Daniel Béland et Jean-Philippe Viriot Durandal, «L'expertise comme pouvoir: le cas des organisations de retraités face aux politiques publiques en France et aux États-Unis », dans Lien social et politiques, $\mathrm{n}^{\circ} 50,2003$, pp. 105-123.

16. Max Weber, Le savant et le politique, Paris, Plon, 1959.

17. Denis Lacorne, L'invention de la république: le modèle américain, Paris, Hachette, 1991, pp. 131-
133.

18. James A. Smith, The Idea Brokers: Think Tanks and the Rise of the New Policy Elite, New York, Free Press, 1991, p. v ; R. Kent Weaver, " The Changing World of Think Tanks », dans PS: Political Science and Politics, septembre 1989, p. 570.

19. Daniel Béland, «Expertise et politique des retraites », op.cit.

20. Pour une perspective historique sur le développement et l'évolution récente des think tanks aux EtatsUnis, on lira Andrew Rich, Think Tanks, Public Policy, and the Politics of Expertise, Cambridge, Cambridge University Press, 2004.

21. L'Heritage Foundation commença à jouer un rôle politique majeur durant les années Reagan. Cette organisation conservatrice fait, aujourd'hui encore, la promotion du libéralisme économique, d'une politique de défense musclée et des valeurs familiales et religieuses traditionnelles.

22. Le CATO Institute défend un libéralisme économique radical fondé sur une philosophie individualiste qui ne met généralement pas l'accent sur les «valeurs traditionnelles» défendues par l'Heritage Foundation et l'American Enterprise Institute, le troisième grand think tank conservateur américain.

23. R. Kent Weaver, « The Changing World of Think Tanks ", op. cit.

24. Dirigée par l'ancien chef de cabinet de l'administration Clinton, John D. Podesta, cette organisation de gauche se réclame explicitement de l'héritage des chefs de file du progressisme américain tells que Teddy Roosevelt, Franklin Delano Roosevelt, John Kennedy et Martin Luther King. Cette organisation soutient notamment une meilleure répartition des richesses et l'instauration d'une couverture universelle maladie aux États-Unis.

25. Andrew Rich, Think Tanks, Public Policy, and the 
Politics of Expertise, op. cit.

26 Pour une discussion détaillée voir Donald E. Abelson et Christine M. Carberry, «Following Suit or Falling behind ? A Comparative Analysis of Think Tanks in Canada and the United States ", dans Canadian Journal of Political Science, n³1(3), 1998, pp. 525-555.

27. Donald E. Abelson, «Any Ideas? Think Tanks and Policy Analysis in Canada », dans Laurent Dobuzinskis, David Laycock et Michael Howlett (dir.), Policy Analysis in Canada: The State of the Art, Toronto, University of Toronto Press, 2007, p. 563.

28. Parmi ces organisations, le Fraser Institute est probablement le plus à droite, et ses positions néolibérales « pures et dures » sont souvent proches de celles du CATO Institute américain. Il est intéressant de noter que le siège social du Fraser Institute se trouve à Vancouver, une ville située à environ 3.500 kilomètres de la capitale fédérale, Ottawa. Les trois autres grands think tanks canadiens mentionnés plus haut (C.D. Howe, CBC et IRPP) sont plus modérés sur le plan idéologique.

29. Donald E. Abelson, Do Think Tanks Matter? Assessing the Impact of Public Policy Institutes, Kingston-Montréal, McGill-Queen's University Press, 2002.
$\mathrm{R} \cdot \mathrm{E} \cdot \mathrm{S} \cdot \mathrm{U} \cdot \mathrm{M} \cdot \mathrm{E}$

Cet article intègre les littératures sur l'impact politique des idées et sur les think tanks afin de mieux saisir le rôle de ces organisations dans l'élaboration des politiques publiques. Après une discussion théorique consacrée aux rapports entre idées, institutions politiques et construction de l'expertise, l'article propose une brève analyse comparative du rôle des think tanks aux États-Unis et au Canada. Cette comparaison explique pourquoi les think tanks jouent un rôle politique plus proéminent aux États-Unis qu'au Canada. Tel que démontré, les institutions politiques américaines favorisent la multiplication des think tanks aux États-Unis. Au Canada, des institutions comme le Bureau du Conseil privé fédéral limitent, au contraire, l'impact politique des think tanks. Une telle situation n'empêche cependant pas les think tanks de jouer un rôle significatif dans l'espace médiatique et politique canadien.

This article bridges the literatures on the political impact of ideas and on think tanks in order to shed more light on the role of these organizations in policy development. After a theoretical discussion devoted to the relationship between ideas, political institutions and construction of expertise, the article formulates a short comparative analysis of the role of think tanks in the United States and Canada. This comparison explains why think tanks play a more prominent political role in the United States than in Canada. As shown, the American political institutions support the multiplication of think tanks in the United States. In Canada, institutions like the federal Privy Council Office limit the policy impact of think tanks. But this situation does not prevent think tanks from playing a significant role in Canadian media and policy debates. 